\title{
Interferometric detection of OAM-carrying Helico-conical beams
}

Engay, Einstom; Bañas, Andrew Rafael; Bunea, Ada-loana; Separa, Stephen Daedalus; Glückstad, Jesper

Published in:

Optics Communications

Link to article, DOI:

10.1016/j.optcom.2018.10.019

Publication date:

2018

Document Version

Peer reviewed version

Link back to DTU Orbit

Citation $(A P A)$ :

Engay, E., Bañas, A. R., Bunea, A-I., Separa, S. D., \& Glückstad, J. (2018). Interferometric detection of OAMcarrying Helico-conical beams. Optics Communications, 433, 247-251.

https://doi.org/10.1016/j.optcom.2018.10.019

\section{General rights}

Copyright and moral rights for the publications made accessible in the public portal are retained by the authors and/or other copyright owners and it is a condition of accessing publications that users recognise and abide by the legal requirements associated with these rights.

- Users may download and print one copy of any publication from the public portal for the purpose of private study or research.

- You may not further distribute the material or use it for any profit-making activity or commercial gain

- You may freely distribute the URL identifying the publication in the public portal

If you believe that this document breaches copyright please contact us providing details, and we will remove access to the work immediately and investigate your claim. 


\section{Accepted Manuscript}

Interferometric detection of OAM-carrying Helico-conical beams

Einstom Engay, Andrew Bañas, Ada-Ioana Bunea, Stephen

Daedalus Separa, Jesper Glückstad

PII: $\quad$ S0030-4018(18)30886-1

DOI: $\quad$ https://doi.org/10.1016/j.optcom.2018.10.019

Reference: OPTICS 23536

To appear in: Optics Communications

Received date: 7 September 2018

Revised date : 5 October 2018

Accepted date : 10 October 2018

Please cite this article as: E. Engay, et al., Interferometric detection of OAM-carrying

Helico-conical beams, Optics Communications (2018),

https://doi.org/10.1016/j.optcom.2018.10.019

This is a PDF file of an unedited manuscript that has been accepted for publication. As a service to our customers we are providing this early version of the manuscript. The manuscript will undergo copyediting, typesetting, and review of the resulting proof before it is published in its final form. Please note that during the production process errors may be discovered which could affect the content, and all legal disclaimers that apply to the journal pertain. 


\section{Interferometric detection of OAM-carrying Helico-conical beams}

Einstom Engay ${ }^{1}$, Andrew Bañas ${ }^{1,2}$, Ada-loana Bunea ${ }^{1}$, Stephen Daedalus Separa ${ }^{2}$ an ' 'esper Glückstad ${ }^{1,2, *}$ ${ }^{1}$ DTU Fotonik, Department of Photonics Engineering, Technical University of Denm גrk, Xrsted Plads 343, DK-2800 Kgs. Lyngby, Denmark

${ }^{2}$ OptoRobotix ApS, DK-2000 Frederiksberg, Der . rrk

*Corresponding author: jesper.gluckstad@foto..", tu.dk

\section{Abstract}

Helico-conical beams (HCBs) are a class of orbital angular momer $n(0, \mathrm{M})$-carrying beams with spiral phase and intensity profiles. In this communication, we demonstrate $t_{1}$. use of a common path interferometer (CPI) to phase-image incoming HCBs and directly detect their OAN associa ed properties. The output intensity of the CPI is a direct mapping of the HCB's phase, hence both th topc oical charge value and sign manifest in the output intensity distribution. The topological charge value is ra. lated by fringe counting, while the helicity is determined by observing the intensity along the $\varphi=0$ res. $n$. With our current CPI setup, we can firmly detect up to $I= \pm 20$. Higher values of $I$ lead to a decrease ir .... sxinge visibility, but this can in principle be improved by tailoring the parameters of the phase contrast fils (PCF) employed in the CPI. We present analytical expressions to optimize the CPI for HCB charge dete $x, n$.

Keywords: Diffractive optics, Physical optics, Inteı, ‘um... ic imaging

Beams with orbital angular momentum $(O A M)$ have attracted much attention in recent years due to their potential applications in optical communir stions '1], high-resolution imaging [2] and optical trapping [3]. OAM arises from helical phased light beams with _ 'imu' hal phase dependence $\exp (i l \varphi)$ [4], where the sign and value of I define the helicity and topological ' nar e (TC) of the beam and $\varphi$ is the azimuthal angle. Such beams include Laguerre-Gaussian (LG) and higher-oru - Bes el beams that carry an OAM equal to $\hbar \hbar$ per photon. A striking feature of these OAM-carrying be $m s$ is tı,e doughnut-shaped far-field intensity profile, which in a classical perspective results from the interferenc of the azimuthal component of the Poynting vectors.

Numerous methods have bee . prr posed to introduce the helical phase to an incident fundamental Gaussian beam. These include emploving 1. . गd phase masks, spatial light modulators (SLMs) and meta-surfaces, amongst others. Of equal importans - fo' most applications is the detection of both the topological charge magnitude and sign of incoming unknown, $7 \mathrm{~m}$. The OAM manifests in the intensity distribution when the beam diffracts through slits and aper ures [ $\ulcorner, 6]$, or when it is made to interfere with another beam [7]. Hence, from the diffraction or interfere ce pat ern, the OAM-content of the beam can be ascertained. Current methods for $I$ determination incl is empruying amplitude diffractive apertures [8-11], interferometers [12-14] and spatial mode projections [15]. $T$, ere has been a recent interest in employing phase-modulating elements because of their efficient deteci ${ }^{-n}$, $f$ the topological charge. Pure-phase gratings have been reported to improve charge detection $[16,1 \%$.

Helico-conical bean. - are a class of OAM-carrying beams whose phase function has a non-separable azimuthal and radial dependence $[18,19]$,

$$
\psi(r, \varphi)=l \varphi\left(K-\frac{r}{r_{0}}\right)
$$


where $K$ is between 0 and 1 , and $r_{0}$ normalizes the radial coordinate $r$. HCBs have both t $v$ isted phase and intensity profiles in the far-field and maintain high photon concentration while still allowin, higher topological charge values. By maintaining high photon densities even at higher values of $I$, HCBs may be suitaw- for applications that require large l's [20]. Particularly, optical communication applications that coul , dı ectly utilize the detected $I$ values are candidates where using a HCB can be beneficial.

The generation of HCBs has already been demonstrated using SLMs [18,211 orir - d fork-type holograms [22] and plasmonic meta-surfaces [23]. Far-field intensity observations have $r$ sen $-u$. Jucted in the past using a $2 f$ lens-configuration where the input HCB phase is encoded on a SLM placen nehind a focusing lens. The spiral profile of the HCB-intensity can be detected by a camera positioned $\mathrm{a}^{+}$the $\mathrm{fc}_{\mathrm{c}}$-al plane of the Fourier lens. Fig. 1 shows examples of HCB intensity profiles for $K=0$ and for different topc ggical, , arge values.
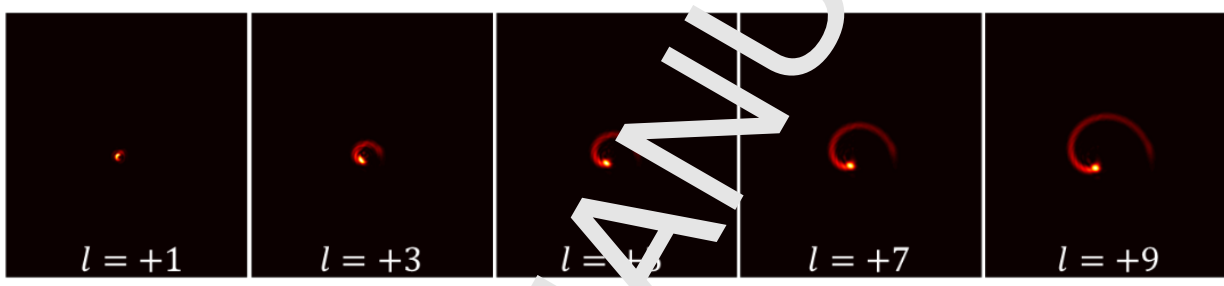

Fig. 1. Spiral far-field intensity profiles of simı 'alc 'Helico-conical beams (HCBs) for $K=0, I=+1$, $+3,+5,+7$ and +9 . The lateral spiral prof _ _.

The propagation dynamics of HCBs have iso bee investigated using far-field intensity measurements $[24,25]$. Although it has been shown that the nten ${ }^{-}+\mathrm{v}$ profiles laterally scale with $I / r_{0}[18]$, the reverse process of determining an unknown topological $c^{\prime}$ arg' of the HCB from the spiral intensities can be a challenging task. This is commonly encountered in optical $\mathrm{co}^{\prime} \mathrm{imu}$. 'r.a' ons, where from one end a transmitter sends out optical beams for data transmission to a receiver on + , nther end for detection and subsequent decoding with no prior information on the incoming beam. HCB topological cr. arge detection from far-field intensity measurements would require all other initial parameters to be $\mathrm{k}^{\prime}$ ow, which is not the case in most OAM-experiments.

In this study, we employ a ' vell-esu hlished common path interferometer (CPI) to image the phase of incoming HCBs and extract their OA' I-as ocis 'ed properties that are otherwise difficult to obtain when observing only their far-field intensity distribıtion, 'i, a $2 f$ lens-setup. We take advantage of the HCB's localized spatial far field intensity distribution $v$ nen $K \quad 0$. A filtering phase element can therefore be reliably aimed at the center of the beam. The higher photc den' ty also ensures that the effective CPI-reference wave has a comparable amplitude compared to the $s$ atial frequency distribution of the HCB being imaged. Had we instead chosen to encode LG beams, the filterir, r eleme nt would coincide with the null region of their ring-shaped profile, resulting in poor contrast, especiallv al w.. her / values. As the CPI provides direct mapping of the HCB phase, the intensity output embodies the tc. $\mathrm{O}^{\mathrm{l}}$ jgıcal charge value and the helicity of the beam. Another advantage of the CPI is its relatively small footprint co. ᄀpared with other interferometry configurations such as the Mach-Zehnder or Michelson interferometers. To optimize HCB charge detection performance, we also analytically examine several parameters that affect the CPI's output contrast. 


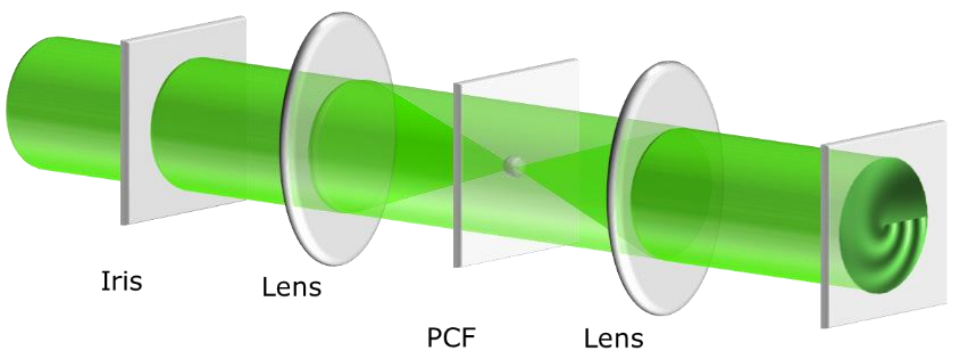

(a)

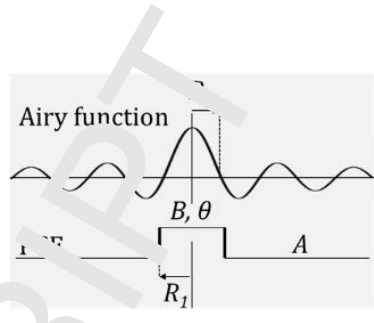

(b)

Fig. 2. (a) Schematic diagram of a $4 f$ common path interferome ter (CPI with a phase contrast filter (PCF) in the optical Fourier plane. (b) PCF parameter veria. un an Airy function, the Fourier transform of a circular input aperture. $A$ and $B$ are ran' . ilt ance factors, $\theta$ is the phase shift introduced by the PCF, $R_{1}$ and $R_{2}$ are the radii of the ${ }^{\circ} \mathrm{Cr}$ and $A^{*}$ y main lobe.

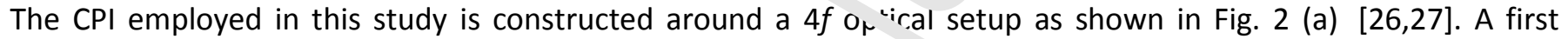
Fourier transforming lens is used to separate the incomir veaun mito a focused light (zero-order) and an off-axis scattered light. A phase contrast filter (PCF) placed at the cen - $^{-}$of the optical Fourier plane is used to impart a $\theta$ phase shift to the focused light, which serves as a v-valled synthetic reference wave (SRW) for the interferometer. The output of the CPI is then the cr'veren superposition of the SRW and the scattered input field that results in the direct mapping of the HCB-phase in $\neg$ ᄂ $\sim$ CPI intensity output. The PCF's transmittance function is given by

$$
\left.H\left(f_{x}, f_{y}\right)=A\left(1+\sin ^{-1} \exp i \theta-1\right) \operatorname{circ}\left(\frac{f_{r}}{\Delta f_{r}}\right)\right)
$$

where $B$ and $A \in[0,1]$ are transmittar ie facu', associated with the focused and scattered light, respectively, $\theta \in[0,2 \pi]$ is the applied phase shift $\circ \mathrm{t}^{\mathrm{t}} \mathrm{e}$ fc -used light within a circular region of radius $\Delta f_{r},\left(f_{x}, f_{y}\right)$ are the spatial frequency coordinates at th Fourier plane and $f_{r}=\sqrt{f_{x}^{2}+f_{y}^{2}}[16,17]$. The filter factors $A, B$ and $\theta$, as depicted in Fig. 2 (b) can be adjus ${ }^{+n}$ accor dingly to improve the visibility of the CPI and for maximum irradiance of the output. With an input $\mathrm{HC}^{\prime}$,-ph se described by the iris-truncated field $U(x, y)=U(r)=\operatorname{circ}\left(r / r_{0}\right) \mathrm{e}^{-i l \varphi r / r 0}$, the intensity at the CPI output berom -

$$
\boldsymbol{I}\left(r^{\prime}\right)=A^{2}\left|U\left(r^{\prime}\right)+\right| \bar{\alpha}\left|\left(B A^{-1}(\exp i \theta)-1\right) g(0)\right|^{2}
$$

where $|\bar{\alpha}|$ is the $\mathrm{nc}$ "malize Fourier zero-order of the input beam, defined as

$$
\bar{\alpha}=|\bar{\alpha}| \exp i \phi_{\bar{\alpha}}=\frac{\int_{0}^{2 \pi} \int_{0}^{r_{0}} U(r, \varphi) r d r d \varphi}{\int_{0}^{2 \pi} \int_{0}^{r_{0}} \operatorname{circ}\left(\frac{r}{r_{0}}\right) r d r d \varphi}
$$

for integer values of $I$. The magnitude of $\bar{\alpha}$ determines the component of the beam that is filtered by the PCF. This parameter vanishes for an LG beam because of its dark center, and thus the CPI would fail to phase-image such a beam. The term describing the $4 f$-imaged object wave, $U\left(r^{\prime}\right)$, in Eq. (3) for $K=0$ is given by 


$$
U\left(r^{\prime}\right)=\operatorname{circ}\left(\frac{r^{\prime}}{r_{0}}\right) \exp \left(-i\left(l \varphi \frac{r}{r_{0}}+\phi_{\bar{\alpha}}\right)\right)
$$

while the central value of the SRW-profile $g(0)$ is [26]

$$
g(0)=g\left(r^{\prime}=0\right) \approx 1-J_{0}(1.22 \pi \eta)
$$

The CPI can also be optimized by tailoring the SRW using the $\eta$ parameter $v \ldots \cdot h$ is c. e ratio of the radius of the filtering region $R_{1}$ to the radius of the mainlobe of the Airy disc $R_{2}$,

$$
\eta=\frac{R_{1}}{R_{2}}=\frac{\mathrm{r}_{0} R_{1}}{0.61 \lambda f}
$$

where $R_{2}$ is written in terms of the input aperture radius $r_{0}$, ill, ....ina $\ldots \ldots$ wavelength $\lambda$ and focal length $f$. Normally, for a circular input aperture, the PCF is fabricated to ma ch + ie 1 adius of the corresponding Airy disc of the Fourier transform distribution. However, it is much more co: 'enient nd practical to use a high quality static filter and, if the application imposes, a specific size on the beam to be '.'tected.

Fig. 3 shows the experimental setup for the CPI. The HCB is oner n_-d using the collimated Gaussian output of a 532-nm diode laser impinging on an SLM (Hamamatsu, $125 \mathrm{um} \mathrm{n.}$. I pitch) which contains the HCB's helical phase distribution. In actuality, the topological charge of the incorı. 'ng HCB is completely unknown from the receiver. In our experiments, however, we generated HCBs witt. .....-11, known topological charge to test our proposed detection scheme. The CPI is constructed using lenses $w^{\text {" }}$, $f=100 \mathrm{~mm}$, and its output is detected with a CMOS camera (Basler, $5.86 \mu \mathrm{m}$ pixel size). Fig. 4 shows the it. רut, ut for different values of $\eta$ and input aperture. In the absence of an encoded phase, the axial alignmen+ $n f$ the PCF can be calibrated so that the expected CPI-output is a dark area as shown in Fig. 4 (a) [28]. With a $\pi$-pha. 2 shifting filter, this is achieved when $K=0.5$ in Eq. (3) that corresponds to $\eta=0.4$ and $r_{0}=1.3 \mathrm{~mm}$ for a PCF radius $R_{1}=9.8 \mu \mathrm{m}$. After the PCF is aligned, the input aperture radius that corresponds to optime iy con, asted HCBs is $r_{0}=2.1 \mathrm{~mm}$, corresponding to $\eta=0.627$. With this value, the Bessel function in Eq. (6) is zt. ? th. SRW and input amplitudes are matched to consequently lead to higher maximum irradiance $I_{\max }$ and visi ility $V=\left(I_{\max }-I_{\min }\right) /\left(I_{\max }+I_{\min }\right)$ within the relevant region of the output intensity distribution. With the $\eta=u_{\Omega^{-}}$) cc idition, Fig. 4 (c) shows the CPI output intensity. In theory, the central intensity value of the outpu' when $\eta=0.627$ is four times the input's central intensity for a $25 \% \pi$-phase fill factor. Fig. 4 (b) and (d) are th $€ \mathrm{CPI}-\mathrm{c}_{\text {. }}$ nuts for a checkerboard input phase pattern for $\eta=0.4$ and $\eta=0.627$, respectively, showing that highe $m_{1}$ ximum intensity and visibility is obtained for the latter.

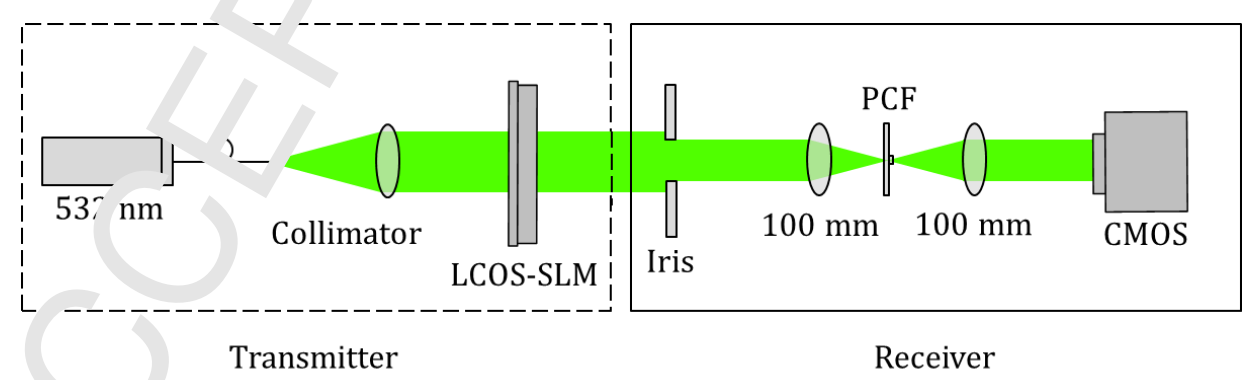

F ?. u. - - merimental setup for HCB detection. An HCB is generated using a 532-nm Gaussian bea. impinging on a LCOS-SLM. The detector or receiver is composed of a CPI interferometer constr .cted using a $4 f$ optical setup. The PCF radius is $9.8 \mu \mathrm{m}$. 


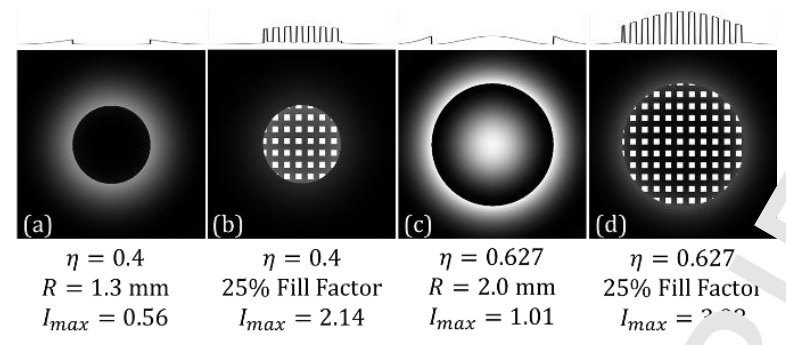

Fig 4. Simulated CPI output for (a), (b) $\eta=0.4$ and (c), (d) $\eta=0.6 .7 . T^{1}$ e dark output in (a) is obtained when the PCF is axially aligned for a circular input aper+*re. " $=0.627$ is chosen to achieve matched amplitude for maximum visibility. (c) is the PI outh it for an input circular aperture with $\eta=0.627$.

Fig. 5 shows the phase-imaged HCBs for $K=0$ and for different, Visual inspection of the boxed region in the images reveals that the number of fringes directly matches the topo sgical charge of the HCB. Given the good output visibility, it would be possible to count these fring - usı.: : nage processing techniques. Scans of each interference pattern such as that in Fig. 6 (a) show that the numb. $r$ of fringes is consistent with the charge for up to $I=20$. It becomes a challenge, however, to determint higher $I$ 's due to the decreased fringe visibility. This highlights the importance of the $\bar{\alpha}$ parameter in Eq. (4)

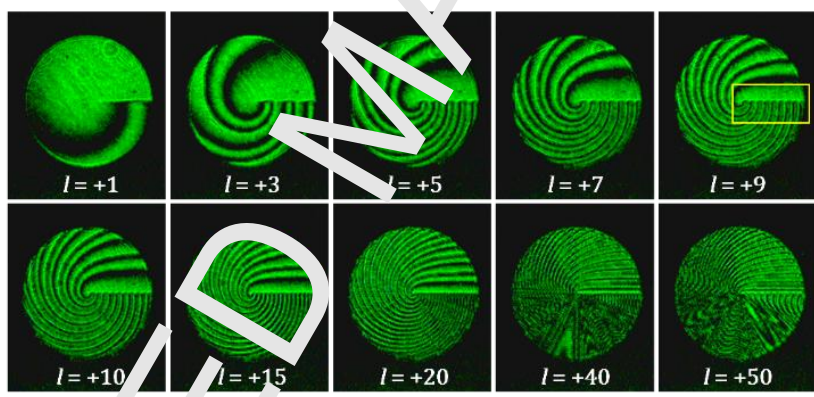

Fig. 5. Experimental ',$C B\left(K=y^{\prime}\right.$ images from the CPI with increasing topological charge.

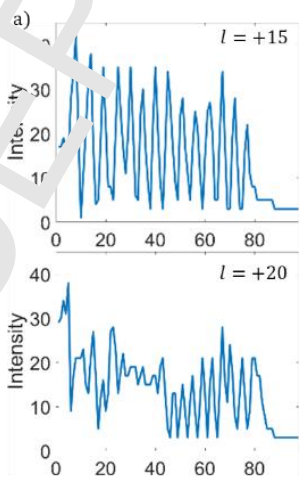

(b)

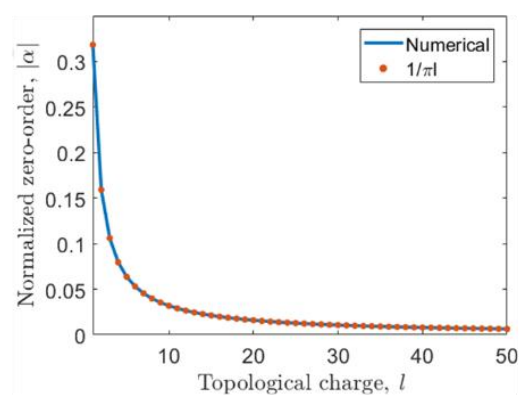

Fig. 6. (a) Ttensity line scans for $I=+15$ (top), +20 (bottom) and (b) normalized zero-order of the HCB with increasing topological charges.

We numerically calculated the normalized zero-order of simulated HCBs and plotted it in Fig. 6 (b) as a function of I. The inverse relation is verified analytically by evaluating Eq. (4) for $K=0, l \neq 0$, leading to 


$$
\bar{\alpha}_{K=0}=-\frac{i}{\pi l}
$$

Thus, from the $|\bar{\alpha}|$ in Eq. (8), the available amount of zero-order for generating a phase-shı. $\sim d$ SRW decreases as $I$ increases. In our current setup, the PCF is based on glass, and hence the filter pa am ters $A$ and $B$ were both $\sim 1$ for a near lossless phase imaging system, allowing us to simplify the expression fo, ${ }^{\text {th }}$. intensity distribution at the output from Eq. (3) into the short form

$$
I_{K=0}=1+\frac{4}{\pi^{2} l^{2}}+\frac{4}{\pi l} \cos l \varphi \frac{r}{r_{0}}
$$

which then leads to the following fringe visibility dependence on $I$ :

$$
V=\frac{4 \pi l}{4+\pi^{2} l^{2}}
$$

The expression in Eq. (9) shows the direct conversion of the HCB's $p_{1}$ use $l e / / r_{0}$ into the CPI output intensity apart from factors that depend on I. However, as described in Eq. (10), tI . ntt-axis scattered light overpowers the SRW for higher I's, resulting in a low fringe visibility. The simple nalytic expression in Eq. (8) can lead to possible further optimization of the CPI, although this is beyond the scon of this Communication. With the use of a dynamic PCF, by using a SLM for example [29], A can bo ani... i so that matched amplitude is still achieved. However, this presents a drawback in possible losses in thc cvstem and a more complex implementation. The presence of moiré fringes due to the limited resolutio. $v_{1}$ - SLM and camera in this demonstration also limits the detection of HCBs with large l's that are not addressan' : with the CPI parameters.

We also examined the output's dependence on the bec $m$ s helicity. Fig. 7 shows the images obtained from the CPI with $I= \pm 1, \pm 2, \pm 4, \pm 10$ and \pm 15 . As describea .' 54 . (J), the intensity distribution of negatively charged HCB's should exhibit fringe shifting with respect to those o, positively charged ones. This is indeed observed in the images acquired in our experiments. The to $\mathrm{vlO}_{5}$. 'al charge information is also preserved in the number of fringes for negative $I$. Moreover, the intensity al ' $\sigma$ the $\rho=0$ region is maximum for positive $I$ (Fig. 7 , top row) and minimum for negative I (Fig. 7, bottom ' Jw). Tr.. Is expected, as described by the intensity distribution in Eq. (9), where we achieve the maximum and nin' nur conditions when $\varphi=0$ for positive and negative $I$, respectively, independent of $I$ and $r$. Hence, by of ,ervin ${ }^{+1}$ e intensity along the $\varphi=0$ region, we can determine the helicity of the incoming $\mathrm{HCB}$.

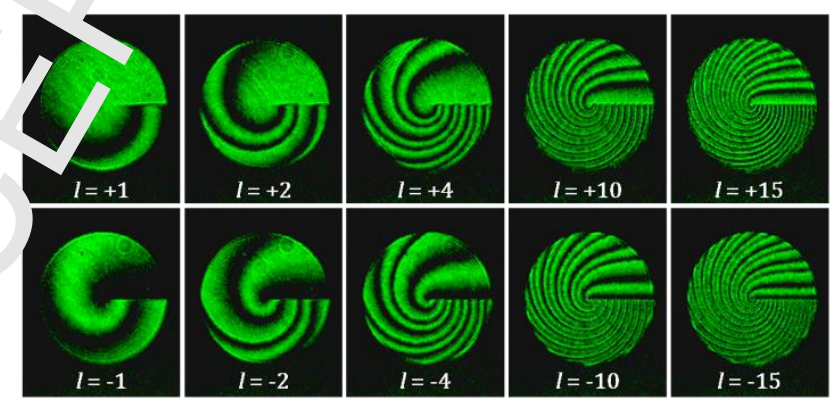

Fig. - (Top row) Positive and (bottom row) negatively charged HCBs $(K=0)$ acquired exper, nentally. A change in helicity manifests itself as a fringe shift at the output of the $\mathrm{CPI}$, with maximum and minimum intensities at $\varphi=0$ for positive and negative $I$, respectively. 
Finally, using the same CPI-setup, we imaged $\mathrm{HCBs}$ with $K=1$ and $K=1 / 2$. Fig. 8 shows the intensity distributions for select topological charges. As with the case of $K=0$, the fringe visibility also decreas - as $/$ increases that in turn leads to the same difficulty of properly identifying the topological charge of the beam for his' 'or orders.

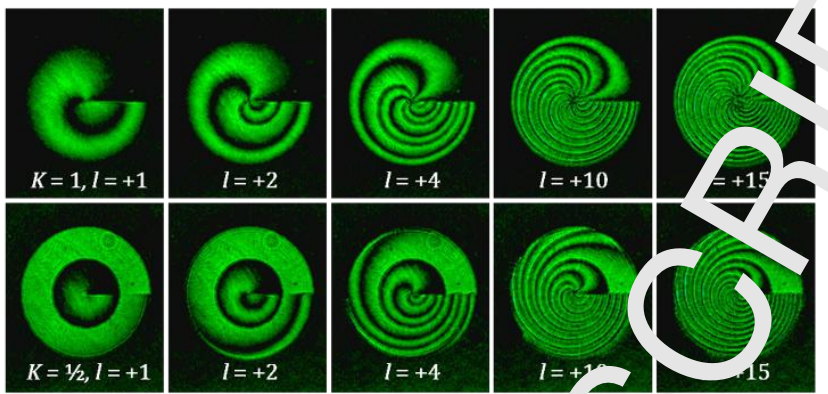

Fig. 8. Experimental CPI output for (top row) $K=+$ and oottom row) $K=1 / 2$.

In conclusion, we have demonstrated the phase imagino of $\mathrm{O}$ - V-carrying HCBs using a common-path interferometer that enables determination of the beam's 'npo, rir ${ }_{\lambda} l$ charge value and its sign. The sufficient photon density around the center in the far-field intensity of HCD, facilitates the formation of a SRW that is phaseshifted by a PCF and can subsequently interfere with the su. ttered light of the input beam. This enables the direct mapping of the HCB-phase at the interferometer's ou'nut intersity embodying both the charge magnitude and sign in the number and behavior of the generated intert. $r$. netric fringes. Using this technique, up to $I=|20|$ can be firmly detected with our current CPI-embodimen. The I. vitation for higher topological charges is mainly due to the weaker fringe visibility associated with a decreasin 8 SkvV-strength for larger topological charges. This could be offset by changing the phase contrast filter param ${ }^{+}$ars iv achieve matched interferometric amplitudes and hence optimal interference. The phase imaging of HCBs with une use of a CPI provides a direct and robust determination of its properties. HCB properties have prev uusıy heen assessed only indirectly, through the analysis of their far field intensities.

Funding. Novo Nordisk Foundation (G' and Challenge Program; NNF16OC0021948). Lundbeck Foundation (Grant number R244-2016-3941).

\section{References}

1. A. E. Willner, H. Huang Y. Y in, Y. Ren, N. Ahmed, G. Xie, C. Bao, L. Li, Y. Cao, Z. Zhao, J. Wang, M. P. J. Lavery, M. Tur, S. Ramac. - Idran, A. F. Molisch, N. Ashrafi, and S. Ashrafi, "Optical communications using orbital angular mom' ntum beums," Adv. Opt. Photonics 7, 66-106 (2015).

2. C. Maurer, A. Jesache, ৎ. ' ernet, and M. Ritsch-Marte, "What spatial light modulators can do for optical microscopy," La' ar Phc+onics Rev. 5, 81-101 (2011).

3. K. Ladavac and $L G$ urier, "Microoptomechanical pumps assembled and driven by holographic optical vortex arrai s," Op’ Express 12, 1144-1149 (2004).

4. L. Allen, M. 、' Ro jersbergen, R. J. C. Spreeuw, and J. P. Woerdman, "Orbital angular momentum of light and the 'a...--mation of Laguerre-Gaussian laser modes," Phys. Rev. A 45, 8185-8189 (1992).

5. Q. S. FerreIı ' A. J. Jesus-Silva, E. J. S. Fonseca, and J. M. Hickmann, "Fraunhofer diffraction of light with orbital angular momentum by a slit," Opt. Lett. 36, 3106-3108 (2011).

6. D. P. Ghai, P. Senthilkumaran, and R. S. Sirohi, "Single-slit diffraction of an optical beam with phase singularity," Opt. Lasers Eng. 47, 123-126 (2009).

7. M. P. J. Lavery, A. Dudley, A. Forbes, J. Courtial, and M. J. Padgett, "Robust interferometer for the routing 
of light beams carrying orbital angular momentum," New J. Phys. 13, (2011).

8. J. M. Hickmann, E. J. S. Fonseca, W. C. Soares, and S. Chávez-Cerda, "Unveili ،g c truncated optical lattice associated with a triangular aperture using light's orbital angular moment. $\cdot m$." Phys. Rev. Lett. 105, 1-4 (2010).

9. C.-S. Guo, L.-L. Lu, and H.-T. Wang, "Characterizing topological charge of op 'ical vortices by using an annular aperture," Opt. Lett. 34, 3686-3688 (2009).

10. L. A. Melo, A. J. Jesus-Silva, S. Chávez-Cerda, P. H. S. Ribeiro, and W. \& c sares, "Direct Measurement of the Topological Charge in Elliptical Beams Using Diffraction by a Trian , uiar Apc ture," Sci. Rep. 8, 1-8 (2018).

11. D. Hebri, S. Rasouli, and M. Yeganeh, "Intensity-based measuring of the + spological charge alteration by the diffraction of vortex beams from amplitude sinusoidal radi „ gratııys," J. Opt. Soc. Am. B 35, 724-730 (2018).

12. H. I. Sztul and R. R. Alfano, "Double-slit interference with - -ouerre-Gaussian beams," Opt. Lett. 31, 1-3 (2006).

13. D. Jianji, Z. Hailong, and Z. Xinliang, "Dynamic interferoı, stry measurement of orbital angular momentum of light," Opt. Lett. 39, 7 (2014).

14. S. Slussarenko, V. D'Ambrosio, B. Piccirillo, . n^arrucci, and E. Santamato, "The Polarizing Sagnac Interferometer: a tool for light orbital angular $\mathrm{m} \sim \mathrm{r}$ ıntum sorting and spin-orbit photon processing," Opt. Express 18, 27205-27216 (2010).

15. A. D'Errico, R. D’Amelio, B. Piccirillo, F. C Jann. and L. Marrucci, "Measuring the complex orbital angular momentum spectrum and spatial mode dec mposition of structured light beams," Optica 4, 1350-1357 (2017).

16. S. Zheng and J. Wang, "Measuring ( rbital A gular Momentum (OAM) States of Vortex Beams with Annular Gratings," Sci. Rep. 7, $40781\left(201^{-}\right)$.

17. Z. Liu, S. Gao, W. Xiao, J. Yang, v Hua Ig, Y. Feng, J. Li, W. Liu, and Z. Li, "Measuring high-order optical orbital angular momentum $v$,th a hy, erbolic gradually changing period pure-phase grating," Opt. Lett. 43, 3076-3079 (2018).

18. C. A. Alonzo, P. J. Rodrig ,, aı d J. Glückstad, "Helico-conical optical beams: a product of helical and conical phase fronts," Opt. Exprc c .3, 1749-1760 (2005).

19. G. Overton and J. G' ^cks .ad, "Phase functions are inseparable in helico-conical optical beams," Laser Focus World (2005).

20. V. R. Daria, D. ¿ Palimc and J. Glückstad, "Optical twists in phase and amplitude," Opt. Express 19, 476481 (2011).

21. T. Latychev kaia ar, Y H. W. Fink, "Inverted Gabor holography principle for tailoring arbitrary shaped threedimensional oam'," Sci. Rep. 6, 26312 (2016).

22. N. P. Heı no a and C. O. Manaois, "Phase structure of helico-conical optical beams," Opt. Commun. 271, 178-183 (2. 77).

23. Y. Zhang, X. Yang, and J. Gao, "Twisting phase and intensity of light with plasmonic metasurfaces," Sci. Rep. 8, 4884 (2018).

24. N. Bareza and N. Hermosa, "Propagation dynamics of vortices in helico-conical optical beams," Opt. Commun. 356, 236-242 (2015). 
1

25. N. Hermosa, C. Rosales-Guzmán, and J. P. Torres, "Helico-conical optical beams self-heal," Opt. Lett. 38, 383 (2013).

26. J. Glückstad and P. C. Mogensen, "Optimal phase contrast in common-path irtorferomıtry.," Appl. Opt. 40, 268-282 (2001).

27. P. J. Rodrigo, D. Palima, and J. Glückstad, "Accurate quantitative phast 'magı $;$ using generalized phase contrast," 16, 2498-2509 (2008).

28. M. J. Villangca, A. R. Bañas, D. Palima, and J. Glückstad, "Dark Gı • - sxtended nodal beam areas from binary-only phase," Opt. Eng. 55, 125102 (2016).

29. F. Kenny, F. S. Choi, J. Glückstad, and M. J. Booth, "Adaptive op misati $n$ of a generalised phase contrast beam shaping system," Opt. Commun. 342, 109-114 (2015). 